\title{
Targeted Radionuclide Therapy: Proceedings of a Joint Workshop Hosted by the National Cancer Institute and the Society of Nuclear Medicine and Molecular Imaging
}

Frederic Fahey ${ }^{1}$, Katherine Zukotynski ${ }^{2}$, Jacek Capala ${ }^{3}$ and Nancy Knight ${ }^{4}$, with input from the Organizing Committee, Contributors, and Participants of the NCI/SNMMI Joint Workshop on Targeted Radionuclide Therapy*

${ }^{I}$ Boston Children's Hospital, Boston, Massachusetts, and Harvard Medical School, Boston, Massachusetts; ${ }^{2}$ Sunnybrook Health Sciences Centre, University of Toronto, Ontario, Canada, and Brigham and Women's Hospital, Harvard Medical School, Boston, Massachusetts; ${ }^{3}$ National Cancer Institute, Bethesda, Maryland; and ${ }^{4}$ University of Maryland School of Medicine, Baltimore, Maryland

$\mathbf{T}$ he growing interest in targeted radionuclide therapy (TRT) for a broad range of applications is shared by a diverse group of medical professionals, including but not limited to physicians and basic scientists in several fields, as well as members of industry, regulatory bodies, and patients. However, no organizational structure is available to regularly bring these stakeholders together to discuss the latest findings and the most productive strategies to ensure that the potential benefits of TRT are realized.

Recognizing the need for a forum to discuss the advances and challenges of TRT relating to availability, supporting technology, and interdisciplinary training and research, the National Cancer Institute (NCI) and the Society of Nuclear Medicine and Molecular Imaging (SNMMI) hosted a joint workshop on March 18 and 19, 2013, at the National Institutes of Health campus in Bethesda, Maryland (1). Plans for the workshop were initiated in 2012 by Dr. Frederic Fahey, SNMMI president, and Dr. Jacek Capala of the NCI, and the 2-d event was cochaired by Dr. Fahey and Dr. Katherine Zukotynski. The event was designed to bring a small but diverse group of stakeholders together (Fig. 1) to discuss contemporary TRT in both structured and open-forum formats, to assess approaches for collaboration, and to evaluate strategies to bring the most promising therapies into routine clinical use. This white paper briefly reviews the discussion on TRT that took place at the workshop and offers next-step recommendations from attendees as summarized in presentations by subject matter experts.

\section{BACKGROUND: RADIONUCLIDE THERAPY, FROM SPECULATIVE TO TARGETED}

Historically, reaching a consensus on the advantages, indications, and implementation of radionuclide therapy, radioimmunotherapy, and TRT has been challenging. In the introduction to MIRD pamphlet no. 22, Sgouros et al. pointed out that the use of ${ }^{224} \mathrm{Ra}$

\footnotetext{
Received Nov. 12, 2013; revision accepted Nov. 15, 2013.

For correspondence or reprints contact: Frederic Fahey, Children's Hospital, 300 Longwood, Ave., Boston, MA 02115.

E-mail: frederic.fahey@childrens.harvard.edu

*These individuals are listed in the Acknowledgment section at the end of this article.

Published online Jan. 6, 2014

COPYRIGHT $\odot 2014$ by the Society of Nuclear Medicine and Molecular Imaging, Inc.

DOI: $10.2967 /$ jnumed.113.135178
}

for cancer therapy was suggested by Alexander Graham Bell as early as 1903 (2,3). However, despite sporadic applications of both $\beta$ and $\alpha$ emitters for clinical oncology proposed during the 20th century, only ${ }^{131}$ I therapy, introduced in 1946 for the treatment of thyroid disease (4), has been consistently used. ${ }^{131}$ I was first used in radioimmunotherapy in 1982 (5), and the first trial of an $\alpha$ emitter in TRT was not reported until 1997 (6). In the past decade, an increasing number of radionuclide and therapeutic agent combinations have been explored in cancer therapy, with a growing body of literature suggesting their effectiveness. The recent U.S. Food and Drug Administration (FDA) approval of ${ }^{223}$ Ra-dichloride for castration-resistant prostate cancer has generated significant interest in $\alpha$ emitters as a strategy for cancer therapy among diverse stakeholders, including patients and their families (7). The working group at the NCI/SNMMI workshop noted that to foster expanding awareness of TRT across the spectrum of medical disciplines, consensus on a single name by which to refer to these therapies is needed. First, it is important to recall that techniques in which a radionuclide is administered with therapeutic intent may or may not be targeted. For example, radionuclide therapy encompasses everything from ${ }^{131}$ I thyroid ablation to localized delivery of theranostic agents, with the term theranostic implying that the agent has potential for both diagnostic and therapeutic use. Radioimmunotherapy describes targeted therapy with radiolabeled monoclonal antibodies. Molecular targeted therapy describes both radionuclide and nonradionuclide therapy (8). TRT describes techniques in which one or more radionuclides, usually but not always incorporated into a conjugate or attached to a ligand, are administered with the goal of providing targeted therapy at the cellular or molecular level.

\section{EXPERT PERSPECTIVES ON THE TRT STATE OF THE ART}

Through a series of presentations, individuals representing different professional perspectives on TRT offered a look at the state of the art and current challenges.

\footnotetext{
A Medical Physicist Perspective

Dr. George Sgouros reviewed the physics of TRT, highlighting the potential of TRT in cancer therapy and emphasizing the role of dosimetry in conducting phase I trials of novel radionuclide agents. TRT delivery, which is not susceptible to the resistance mechanisms seen with chemotherapy, kills targeted cells instead of inhibiting growth or survival pathways and precludes adaptation. Further, TRT can be paired with imaging and other techniques to predict
} 


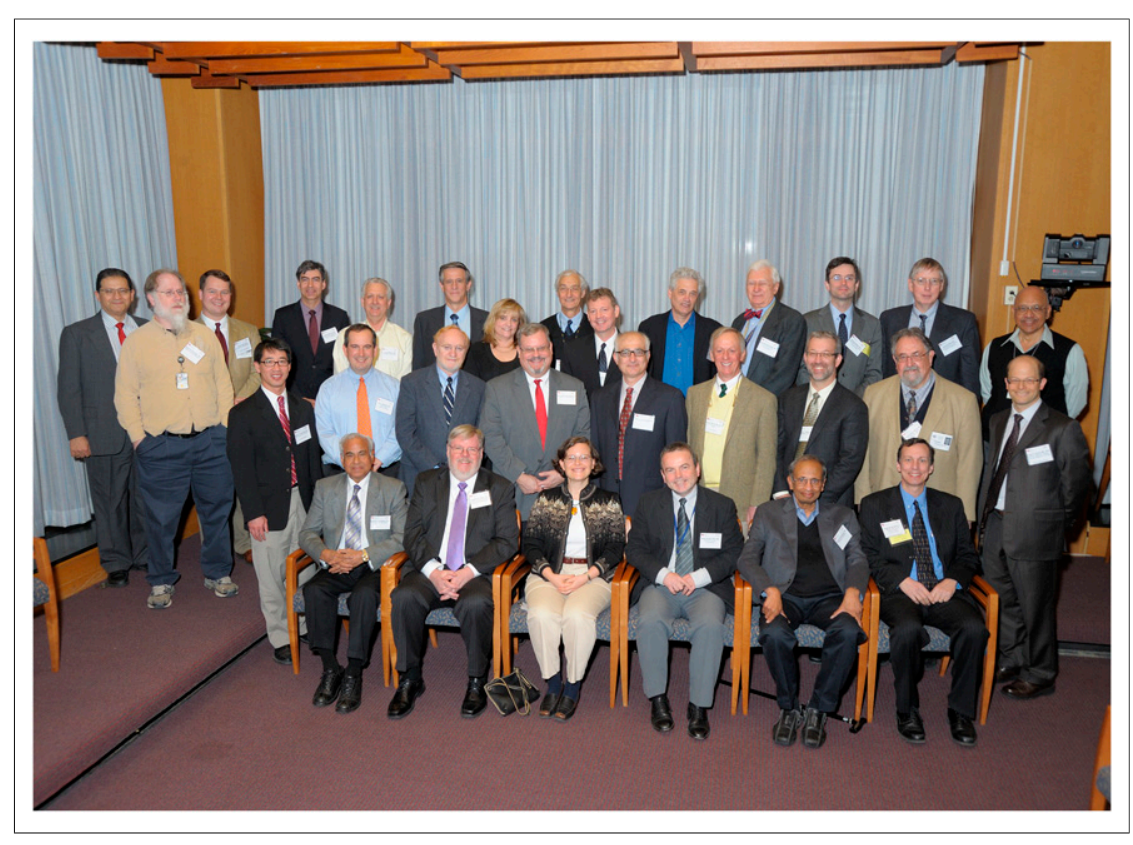

FIGURE 1. More than 40 molecular imaging physicians, physicists, oncologists, basic scientists, and others gathered at the National Institutes of Health on March 18 and 19, 2013, for a workshop on targeted radionuclide therapy.

delivery of therapeutic agents to tumor targets and normal organs before therapy administration and thus can be used to guide escalation protocols and treatment planning. These advantages make TRT especially promising in several cancers.

Dr. Sgouros stressed the importance of reassessing dosimetry methods used in TRT, emphasizing the need to adopt methods specific to the therapy being used and geared to evaluating treatment efficacy and toxicity. Current dosimetry methods associated with and appropriate to radioimmunotherapy, for example, are often model-based methods for risk evaluation. In general, these methods are not patient-specific, do not account for nonuniformity, and do not predict toxicity or efficacy. In the context of TRT, Dr. Sgouros recommended moving away from average absorbed dose, noting that a single absorbed dose volume is useful only if it successfully predicts biologic effects. He recommended adoption of patient-specific, 3-dimensional (3D) dosimetry coupled with radiobiologic modeling, where $3 \mathrm{D}$ internal dosimetry has the advantages of being patient-specific, using accurate anatomy and activity data, calculating absorbed dose voxel by voxel, and providing output dose as a mean over a chosen volume or dose-volume histogram (9-11). Also, 3D radiobiologic dosimetry with Monte Carlo calculations and radiobiologic modeling to predict response from absorbed dose can produce a more faithful prediction of tumor response and toxicity to normal tissue (12-14). Dr. Sgouros provided examples of 3D radiobiologic dosimetry capabilities in clinical cases, as well as comparisons with organ mean absorbed doses that were calculated with OLINDA software (15). He pointed to the general agreement of these methods and the advantages of $3 \mathrm{D}$ radiobiologic dosimetry in real-time treatment planning with patient-specific dosimetry and the potential for additional utility in combined-modality therapy (16).

Dr. Sgouros reviewed considerations for $\alpha$-particle dosimetry and characteristics that distinguish it from $\beta$-particle therapy. For example, with $\alpha$-particle therapy, the mean dose to any macroscopic target volume may not predict overall biologic effect (i.e., some cells may receive no dose, whereas others receive a high dose), such that total effective dose depends on the spatial distribution of activity relative to the target cell population. He illustrated this principle with ${ }^{223} \mathrm{Ra}$ bone dosimetry for the treatment of castration-resistant prostate cancer (with the newly approved agent Xofigo [ ${ }^{223}$ Ra-dichloride; Bayer HealthCare Pharmaceuticals]) (17) and with ${ }^{213} \mathrm{Bi}$-labeled monoclonal antibodies in mouse models of breast cancer metastases (18). As in other areas of TRT research, pairing preclinical studies with human studies allowed derivation of microscale absorbed dose without human autoradiography. As part of the translation of these studies to clinical use, Dr. Sgouros suggested including absorbed dose as an escalation variable in phase I clinical studies.

Dr. Sgouros concluded his talk by emphasizing the importance of collaboration among various stakeholders in the TRT process to ensure its advancement, citing Mercadante and Fulfaro (19): "The decision to use radiopharmaceutical agents should be based on a multidisciplinary plan, involving radiation oncology, nuclear medicine, and medical oncology."

\section{A Radiochemist Perspective}

Dr. Michael R. Zalutsky summarized the current status of the radiochemistry underlying TRT. The critical importance of appropriate radionuclide selection to the success of TRT was stressed. Also, a variety of key considerations were discussed, including the need for radionuclide availability at reasonable cost and selection of an appropriate labeling methodology.

Dr. Zalutsky emphasized that improved labeling methodologies would result in more specific and selective tumor targeting and therapeutic efficacy. Criteria to be considered in selecting a labeling method included the need for specific activity, preservation of biologic function, stability in vivo, adaptability to high activity levels, and characteristic tumor-mediated degradation. In a detailed summary of current labeling strategies, Dr. Zalutsky reviewed the process of labeling radiometals (acyclic chelated [diethylenetriaminepentaacetic acid, or DTPA] and macrocycles [DOTA]) and radiohalogens (direct electrophilic [IODO-GEN; Pierce Biotechnology, Inc.] and prosthetic group conjugation [Bolton-Hunter, dehalogenation resistant, and residualizing]). Several challenges associated with conventional radiosynthesis of radiolabeled agents were discussed, and the solid-phase synthesis of ${ }^{211}$ At-meta-astatobenzylguanidine was presented as an example of a simpler radiochemical approach more suited to routine use. Of note, specific activity poses greater difficulties in TRT than in diagnostic imaging-with diagnostic imaging, acceptable contrast is needed, whereas with TRT, homogeneous delivery of sufficient radionuclide to a target is needed to have the desired therapeutic effect. In a study by Akabani et al. (20), although ${ }^{211}$ At-labeled trastuzumab monoclonal antibody was shown to be effective for treating HER2-positive tumor cells, the specific activity of the agent was an important variable influencing the efficiency of cell killing. The challenges in TRT are compounded by the fact that some molecular targets are expressed at low levels 
and most have a high degree of heterogeneity within individual tumors.

Dr. Zalutsky noted the importance of understanding radiotherapeutic agent metabolism in normal tissues and tumors, as well as the nature and disposition of labeled catabolites that are generated, in order to optimize therapy and reduce toxicity. Considerations regarding the biodistribution of labeled catabolites were illustrated with ${ }^{177} \mathrm{Lu}$ - and ${ }^{125} \mathrm{I}$-labeled agents, and tumor-to-tissue ratios using bone, liver, tumor, blood, spleen, thyroid, and kidneys were shown. Methods for accurately assessing internalization into specific molecular targets were discussed for both research and clinical applications.

Also discussed was an additional factor that can confound radiochemistry at therapeutic dose levels: the effect of radiolysis. Radiation can generate ions, free radicals, and other molecules in a complex process yielding a spectrum of products dependent on type of radiation, dose rate, and presence of trace components. This was illustrated using ${ }^{211} \mathrm{At}$, where the effects of radiolysis can result in lower labeling yields at higher activity levels and at varying times of day, and poor reactivity in ${ }^{211}$ At shipped from one site to another. Lack of reliability in the preparation of clinical doses larger than $350 \mathrm{MBq}$ and challenges to commercialization were reviewed. The need to develop approaches to meet these challenges were underlined and illustrated using the synthesis of $\mathrm{N}$-succinimidyl 3-211 At-astatobenzoate for preparation of clinical doses of ${ }^{211}$ At-labeled radiopharmaceuticals.

Dr. Zalutsky concluded his talk by suggesting that labeling methods are currently available for most radionuclides of clinical interest for TRT. However, concerted efforts will need to be made to ensure production of labeled compounds at activity levels sufficient for TRT and to address challenges associated with specific activity, radiolysis, and catabolism to maximize effectiveness and safety.

\section{A Nuclear Medicine Physician Perspective}

Dr. Richard L. Wahl provided an overview of the history and emerging opportunities in TRT. He began with a review of ${ }^{131} \mathrm{I}$ therapy in thyroid cancer $(21)$ and the use of ${ }^{124} \mathrm{I}$ PET/CT as a guide in the diagnosis and treatment of tumors with $B R A F$-activating mutations (22). Dr. Wahl then provided an in-depth look at the clinical use of tositumomab and ${ }^{131}$ I-tositumomab (Bexxar; GlaxoSmithKline), the radiolabeled anti-CD20 monoclonal antibody approved for the treatment of follicular lymphoma. Follicular non-Hodgkin lymphoma (NHL) is the second most common type of NHL and accounts for $25 \%-40 \%$ of all adult lymphoma. The overview included a discussion of CD20 expression in the B-cell life cycle, characteristics and mechanisms of action of the constituent radiolabel and monoclonal antibody, and the treatment regimen currently used $(23,24)$. Dosimetry considerations for ${ }^{131} \mathrm{I}$-tositumomab treatment were reviewed, and the need for an accurate and personalized approach to maximize radiation dose to tumor and minimize patient toxicity was underlined. The concept was discussed that dosimetry studies confirm great variation in antibody clearance among patients, depending on factors such as tumor size, splenomegaly, or the amount of bone marrow involvement. The importance of adjusting the amount of radioactivity given to ensure all patients individually receive a total body dose of $75 \mathrm{cGy}$ was emphasized. The advantages of careful dosimetry calculations allowing physicians to prospectively individualize therapeutic dose were reviewed. The rationale was discussed for administering unlabeled tositumomab before the ${ }^{131}$ I-labeled antibody, along with the results of clinical studies of ${ }^{131} \mathrm{I}$-tositumomab in patients treated with rituximab. Data were presented suggesting benefits of ${ }^{131}$ I-tositumomab retreatment in patients who previously responded to ${ }^{131} \mathrm{I}$-tositumomab therapy (25). The fact that ${ }^{131}$ I-tositumomab has been shown to have an overall response rate of $95 \%$ (complete response, $75 \%$ ) in patients with previously untreated, advanced-stage, low-grade NHL was discussed (26). Studies of up-front radioimmunotherapy plus chemotherapy, the use of ${ }^{131}$ I-rituximab radioimmunotherapy in relapsed or refractory indolent NHL (27), and the use of rituximab versus $\mathrm{CHOP}$ chemotherapy (cyclophosphamide, hydroxydaunomycin, vincristine, and prednisone) plus ${ }^{131}$ I-tositumomab in newly diagnosed follicular NHL (28) were reviewed.

The mechanism of action and therapeutic regimen for ${ }^{90} \mathrm{Y}$ ibritumomab tiuxetan (Zevalin; Spectrum Pharmaceuticals, Inc.), a monoclonal antibody TRT for relapsed or refractory, low-grade follicular or transformed B-cell NHL, was presented. It was mentioned that response rates for ${ }^{90} \mathrm{Y}$-ibritumomab tiuxetan were better than those for rituximab alone in phase III trials (29). Also, it was suggested that recent follow-up to the multicenter first-line trial of ${ }^{90}$ Y-ibritumomab tiuxetan as consolidation therapy for first remission in advanced-stage follicular NHL indicates high overall and complete response rates (30). However, although ${ }^{90}$ Y-ibritumomab tiuxetan appears to be well tolerated and treatment can be given on an outpatient basis without radiation-related lifestyle restrictions, its toxicity may be dose-limiting and proper patient selection remains an important part of clinical practice. Variability in toxicity, response, and organ dose has been identified but not yet entirely explained. Methods to account for these variabilities and accurately estimate delivered activity are under investigation, including the use of ${ }^{111} \mathrm{In}$-based organ dosimetry (31).

Current challenges in radiolabeled somatostatin analog therapy were then discussed, including the question of whether to administer small empiric doses without prior dosimetry calculations or to use dosimetry calculations to modulate renal dose (32). The importance of dose escalation studies in answering these questions was emphasized $(33,34)$. Also briefly discussed were the growing use of ${ }^{90}$ Y-labeled microsphere and particle treatment for liver metastases and primary tumors and FDA-approved therapies for bone metastases, including ${ }^{153} \mathrm{Sm}$-EDTMP (ethylenediaminetetramethylene phosphoric acid), ${ }^{89} \mathrm{Sr}$, and, most recently, ${ }^{223}$ Ra-dichloride.

In summarizing the current outlook for TRT, Dr. Wahl suggested that an overall limitation is the need to ensure accurate delivery of radiopharmaceutical to the target and that despite evidence suggesting TRT is effective as a single agent, combination of TRT with additional anticancer therapy may be essential to curative approaches. Dr. Wahl emphasized that collaborative work is needed to integrate TRT into standard oncologic treatment regimens (35), an effort that will require a cadre of knowledgeable health-care professionals. Dr. Wahl also stressed that personalized dosimetrybased therapy is likely to become increasingly important in TRT and that economic concerns, including risks associated with developing new agents and reimbursement issues, will need to be addressed for TRT to become a viable option. Finally, the need for exploration of different targets and delivery methods was emphasized, and an effort to create well-defined TRT funding mechanisms and collaborative trial groups was proposed.

\section{A Medical Oncologist Perspective (Lymphoma)}

Dr. Eric Jacobsen discussed the role of radioimmunotherapy in lymphoma with emphasis on follicular lymphoma, diffuse large B-cell lymphoma, and mantle cell lymphoma. A thorough overview of clinical trials in these disease settings was provided (36-41). 
The effectiveness of the anti-CD20 murine monoclonal antibodies ( ${ }^{90}$ Y-ibritumomab tiuxetan and ${ }^{131}$ I-tositumomab) in relapsed or refractory follicular lymphoma was reviewed. It was noted that both agents are FDA-approved (2002 and 2003, respectively), and their advantages as single-agent, front-line therapy when compared with rituximab were discussed $(26,42,43)$. The challenges faced by medical oncologists considering ${ }^{90}$ Y-ibritumomab tiuxetan or ${ }^{131}$ I-tositumomab therapy for patients with follicular lymphoma were also mentioned. The fact that medical oncologists frequently report concerns about bone marrow damage and late side effects associated with therapy, as well as a general preference for nonradioactive treatment alternatives, was discussed. Practical concerns were emphasized, including difficulty finding an appropriate site for patient referral, the complexity of the referral process, and the fact that referring patients to another physician for treatment may adversely affect the referring practice's bottom line. Dr. Jacobsen also noted that a compelling barrier to the use of ${ }^{90}$ Y-ibritumomab tiuxetan or ${ }^{131}$ I-tositumomab from a medical oncologist perspective is the lack of definitive randomized clinical trial data showing therapy effectiveness and improved outcome. The available studies, he suggested, leave unanswered questions about the role of radioimmunotherapy in first-line or consolidation treatment for follicular lymphoma $(43,44)$.

In diffuse large B-cell lymphoma, although radioimmunotherapy has met with success as consolidation therapy after rituximab/ CHOP in high-risk, untreated disease $(45,46)$, recent trials comparing rituximab/BEAM (carmustine, etoposide, cytarabine, and melphalan) versus ${ }^{131}$ I-tositumomab/BEAM in relapsed diffuse large B-cell lymphoma after autologous stem cell transplantation found no difference in progression-free or overall survival (47). Dr. Jacobsen summarized his review of trials on diffuse large Bcell lymphoma by defining current barriers to implementation of radioimmunotherapy. Indeed, radioimmunotherapy may be contraindicated or less effective in patients with extensive marrow infiltration, cytopenia, or bulky disease. Currently available data on the use of radioimmunotherapy in upfront consolidation are not definitive and in some cases are negative. Further, patients may be unwilling to face intervention before ${ }^{90}$ Y-ibritumomab tiuxetan or ${ }^{131}$ I-tositumomab therapy such as the need for a bone marrow biopsy. Also, there remains concern about the effect of radioimmunotherapy on the potential range of future treatments.

Dr. Jacobsen concluded his review with a brief look at current research on the potential for radioimmunotherapy as consolidation therapy or as preconditioning in mantle cell lymphoma $(48,49)$.

\section{A Medical Oncologist Perspective (Bone Disease)}

Dr. David I. Quinn reviewed past and current TRT in bone disease. In metastatic castration-resistant prostate cancer, treatment options are again proliferating after a hiatus from about 2004 to 2010. The spectrum of therapy available for prostate cancer was reviewed, including the recent development of several novel agents including immunotherapy, cytotoxic chemotherapy, rank-ligand inhibitors, and agents targeting the androgen-receptor pathway. In the midst of this dynamic milieu, level 1 clinical evidence of benefit for TRT has emerged, along with unanswered questions regarding optimal treatment selection and sequence of therapy (50-59).

Dr. Quinn reviewed new treatments, with a focus on current and recently completed trials $(57,60,61)$. He noted that the use of radionuclides in bone disease is not new. Both strontium and samarium have been in use for more than a decade. For each trial cited, Dr. Quinn reviewed the historical precedents. He gave special attention to data from the Alpharadin in Symptomatic Prostate Cancer Patients (ALSYMPCA) trial, a phase III study of ${ }^{223} \mathrm{Ra}-$ dichloride in men with symptomatic castration-resistant prostate cancer and skeletal metastases sponsored by Bayer HealthCare Pharmaceuticals and Algeta ASA, and opened in 2008 (62). The aim of the study was to compare the efficacy of standard of care plus ${ }^{223} \mathrm{Ra}$-dichloride versus standard of care plus placebo. The primary efficacy endpoint was overall survival, and secondary endpoints included evaluation of total serum alkaline phosphatase and prostate-specific antigen levels. The ALSYMPCA trial's rationale was based on numerous studies suggesting that ${ }^{223} \mathrm{Ra}$ targets areas of new bone formation and metastases, with highly localized cell killing and minimal damage to surrounding normal tissues $(63,64)$. Results indicate that in men with castration-resistant prostate cancer and bone metastases, ${ }^{223}$ Ra-dichloride significantly improved survival (median, 14.9 mo vs. $11.3 \mathrm{mo}$; hazard ratio, 0.70; 95\% confidence interval, $0.580 .83 ; P<0.001)$ and median time to first skeletal related event compared with standard of care (62). ${ }^{223} \mathrm{Ra}$-dichloride was also associated with low myelosuppression rates and fewer adverse events.

Dr. Quinn concluded by suggesting that TRT will soon become a mainstay of prostate cancer treatment, with opportunities for expansion to other cancers, such as breast, lung, and primary bone tumors, as well as in myeloma. Dr. Quinn also suggested the need for treatment algorithms leading to a personalized therapy approach in men with castrate-resistant disease.

\section{A Medical Oncologist Perspective (Neuroendocrine Tumors [NETs])}

Dr. Jorge Carrasquillo provided an overview of radionuclide therapy in NETs, a diverse group of diseases with widely varying characteristics requiring different therapeutic approaches. Dr. Carrasquillo provided a comprehensive review of the current status of research in this area. He discussed the role of ${ }^{131}$ I-metaiodiobenzylguanidine ( $\left.{ }^{131} \mathrm{I}-\mathrm{MIBG}\right)$, which was first used in the early 1980 s to target the noradrenaline receptor (65), and he reviewed a variety of NETs that express this receptor and have been targeted for imaging and therapy. The literature of ${ }^{131}$ I-MIBG therapy in patients with paraganglioma, pheochromocytoma, and neuroblastoma was discussed (66-85), and TRT in carcinoid and medullary thyroid cancer was reviewed. Dr. Carrasquillo also reviewed patient preparation, protocols for TRT, and response criteria assessment across the spectrum of disease. Other topics included combination of TRT with chemotherapy or sensitizing agents, intraarterial TRT administration, pretherapy imaging providing quantitation of therapeutic delivery, therapy regimens varying in specific activity and dose, and the potential for astatine-labeled MIBG.

Somatostatin receptors in NETs, particularly in carcinoids and gastroenteropancreatic tumors, were discussed with emphasis on the use of ${ }^{111} \mathrm{In},{ }^{90} \mathrm{Y}$, and ${ }^{177} \mathrm{Lu}$ labels and theranostic agents. Somatostatin receptors, including the 5 subtypes (1, $2 \mathrm{a}$ and $2 \mathrm{~b}, 3,4$, and 5) have been well described in the literature, and numerous studies have reported on the characteristics and affinities of radiolabeled somatostatin analogs, including ${ }^{111}$ In-DTPA-octreotide (Octreoscan; Mallinckrodt Pharmaceuticals), ${ }^{90} \mathrm{Y} /{ }^{177} \mathrm{Lu}$ DOTATOC, ${ }^{177} \mathrm{Lu} /{ }^{90} \mathrm{Y}$ DOTATATE, and ${ }^{90} \mathrm{Y}$ DOTA-lanreotide $(86,87)$. Studies have addressed response rates, outcomes, the role of imaging (particularly $\mathrm{PET} / \mathrm{CT}$ with ${ }^{68} \mathrm{Ga}$-DOTATOC) in guiding therapeutic management, and the relative effectiveness of different radiolabeled somatostatin receptor agents (88-107). Concerns about renal toxicity were discussed, and the use of dose fractionation, amino acid infusions, 
radioprotectants, and other mitigating approaches were reviewed as a way to lessen adverse effects.

Dr. Carrasquillo concluded that although both ${ }^{131} \mathrm{I}-\mathrm{MIBG}$ and radiolabeled somatostatin analogs have shown benefits in symptomatic management and disease response, the impact of study data on clinical practice has been limited for a variety of reasons, including in part the relatively low incidence of target diseases. The need for future investigation to identify optimal peptides and chelates, develop practical and effective therapy protocols, establish dosimetry models, and determine which diagnostic imaging agents are best suited to specific therapy was touched on. Dr. Carrasquillo noted that each of these concerns should be addressed through well-designed clinical trials.

\section{A Radiation Oncology Perspective}

Dr. Bhadrasain Vikram reviewed the complementary role of TRT in radiation oncology. He began by reminding attendees that radiation oncologists attempt to deliver radiation doses as high as possible to malignant targets while avoiding toxicity to nonmalignant tissues. External-beam (teletherapy) and implanted solid radioactive sources (brachytherapy) are the 2 most common delivery approaches. However, the challenge remains that the ability to distinguish malignant from nonmalignant tissues is key and often difficult. Further, distinguishing these tissues may be complicated by the presence of occult metastases. The use of diagnostic and therapeutic radionuclides and radiopharmaceuticals can complement both teletherapy and brachytherapy. For this to be practical, however, improved targeting and labeling methods, optimization of radionuclide agents, and improved dosimetry are urgently needed such that TRT will be accepted as a routine adjunct to clinical radiation oncology practice.

Dr. Vikram discussed next-generation characteristics of teletherapy and the need for accurate identification of subvolumes that would benefit from high radiation doses versus those that would not. He identified the disciplinary tasks that would be required to create and disseminate the next generation of TRT in radiation oncology practice. From a basic sciences perspective, he stressed that these include identification of optimal radionuclides, refinement of microdistribution-based treatment planning, and development and commercialization of new delivery and supporting technologies. Careful attention to radiolabeling techniques for new and evolving tumor-targeting agents is needed, and identification and characterization of new tumor-specific targets, biomarkers, and radiosensitizers is important. Validation using preclinical and translational studies will be essential. To be adopted into clinical practice, clinical trials must be carefully planned and initiated, and tumor TRT will need to be incorporated or compared with current treatment strategies.

\section{CONSENSUS FROM MULTIDISCIPLINARY WORKSHOPS}

Four breakout discussion sessions were held as part of the NCI/SNMMI workshop. In these sessions, cross-sections of stakeholders were asked to focus their discussion on one of the following topics: lymphoma, bone therapy, solid tumors, and NETs. Summaries of the discussions and consensus reached by each group were presented with a focus on current TRT state of the art, clinical indications, apparent strengths and weaknesses, the most promising advances in the field, and challenges to overall acceptance and incorporation into routine clinical use. Despite the spectrum of diseases discussed, the groups shared several similar conclusions.

\section{Lymphoma}

Dr. Janis O'Malley summarized the results of the breakout group focusing on lymphoma. The group identified, among other long- and short-term objectives, the need for evidence-based clinical trials to generate robust data on radioimmunotherapy and TRT effectiveness, the utility of developing centers of excellence to coordinate and translate advances from basic science to clinical use, and the benefit of increased interaction across the spectrum of stakeholders in targeted therapy.

The group commented on the limited use of radioimmunotherapy in routine clinical practice today, now more than a decade after initial FDA approval of radioimmunotherapy for relapsed or refractory low-grade or follicular NHL and the expansion of approval in 2009 to first-line radioimmunotherapy in previously untreated follicular NHL with partial or complete response to chemotherapy. Although data on ibritumomab and tositumomab in lymphoma suggest radioimmunotherapy is well tolerated and may be superior to rituximab alone, many of the prior clinical trials were single-arm studies or compared radioimmunotherapy with chemotherapeutic agents that are now obsolete. Moreover, the poor cure rate of aggressive, transformed low-grade lymphoma has contributed to skepticism among referring clinicians about the true extent of radioimmunotherapy benefit. Several participants suggested there was a need for additional data from well-designed clinical trials to advance acceptance and widespread use of radioimmunotherapy in lymphoma. Such trials, it was thought, would ideally compare radioimmunotherapy with current chemotherapeutic strategies and provide data on cost effectiveness, quality-of-life benefit, progression-free survival, and overall survival. It was recognized that a major challenge to developing these trials would be the dynamic nature of cancer treatment since therapies are rapidly evolving and may have changed by the time of study completion. Trial design and selection of the targeted patient pool were thought to be critical as well. The careful selection of patient groups most likely to benefit from radioimmunotherapy would likely expedite therapy to those in most need. Identification of underserved patient groups, genomic and individual characteristics, and development of multicenter collaborative initiatives to expedite statistically significant findings would be helpful. Patients most likely to benefit from radioimmunotherapy were thought to include the elderly and those with difficult-to-treat disease (e.g., mantle cell lymphoma and diffuse large B-cell lymphoma with activated B-cell monotype).

One challenge to increasing radioimmunotherapy acceptance and use in lymphoma is that current ${ }^{90} \mathrm{Y}$ - and ${ }^{131} \mathrm{I}$-labeled CD20targeting agents are not sufficient for the range of diseases. The group suggested that researchers should consider looking beyond CD20 as a target in NHL to applications in Hodgkin lymphoma and T- and B-cell NHL. Further, the logistics of current radioimmunotherapy use were seen as a barrier to growth. The field has no academic or research home-in various institutions radioimmunotherapy practice may be centered in radiation oncology, nuclear medicine, hematologic oncology, or general oncology, with research distributed among basic scientists and health-care professionals. Personnel trained in radioimmunotherapy are difficult to find, and champions are few, even in many academic centers. Current protocols and referral processes that are often disruptive to routine clinical practice may offer obstacles in terms of stakeholder interest and reimbursement issues.

The group suggested that to increase radioimmunotherapy use, dissemination of knowledge across multiple disciplines, implementation of easily available and useable agents, appropriate reimbursement, 
and the support of well-trained and enthusiastic physicians and dedicated personnel are needed. Agents with new cell targets, minimal side effects, and novel carrier molecules would be of benefit. Studies exploring personalized dosimetry remain a critical issue.

The discussion group recommended a collaborative approach, both within and among institutions, in which basic researchers and clinicians would regularly meet and assess strategies to investigate new targets, labels, patient populations, and regimens. The group cited as an example the potential of various radiolabels (e.g., ${ }^{211} \mathrm{As}$ and ${ }^{225} \mathrm{Ac}$ ) that have shown promising characteristics for radioimmunotherapy. Sites with the capability of developing, validating, and evaluating these agents both preclinically and clinically could play a central role. It was suggested that the creation of a network of centers of excellence that could pool expertise and findings would be helpful.

One of the overarching needs is to bring patients more actively into the focus of TRT research. Many patients are afraid of radiation or may not be willing to undergo the work-up needed for TRT. Studies providing compelling evidence that TRT improves outcomes-with lower toxicity than chemotherapy - are needed, preferably including validated metrics of patient-reported outcomes and toxicities. To facilitate the range of steps needed to advance understanding and use of TRT, the group made several short- and longer-term recommendations.

In the short term:

- The SNMMI should reach out to various societies such as the American Society of Clinical Oncology and the American Society of Hematology and invite them to scientific meetings and special sessions to promote collaboration.

- A lymphoma summit meeting, modeled on this workshop, including scientists, clinicians, and support personnel, would be helpful in identifying low-hanging fruit in TRT and creating collaborations among researchers across institutions.

- Collaborative trials focused on underserved populations or new targets and agents should be formed. In particular, the group suggested that an interdisciplinary panel might plan a new collaborative national trial on ibritumomab or tositumomab.

In the longer term:

- A working group should be formed either to identify an existing nationwide trial network or to support creation of a new network to coordinate nationwide centers of excellence in TRT to jointly explore advances from basic sciences to clinical implementation. This would include development of multicenter protocols and trials.

- Increased outreach and training should be emphasized. Clinicians and investigators with the expertise, training, and tools to advance radioimmunotherapy are essential to sustain forward motion in the field. It was recommended that work with residency review committees across specialties be done to promote inclusion of TRT in training. Maintenance-ofcertification programs could also be used as a tool to engage both those who are already involved in TRT and those who have an interest, and these programs could be offered both within and outside the traditional core of nuclear medicine-oriented professional meetings. Certification examinations could also be amended to include TRT as a requisite part of training.

\section{Bone Disease}

Dr. David Quinn presented the results from the breakout group focusing on bone therapy, summarized factors affecting current and future standards of care, and discussed key questions for future clinical trials. The importance of fostering cooperative groups was emphasized.

Dr. Quinn suggested that current treatment of bone metastases is changing, as is our understanding of the science that underlies it. The availability of new oral hormonal agents, for example, may mean that patients will remain longer under a urologist's care and that the urologist will be required to interact with other specialists to obtain immunotherapy or radionuclide treatments for patients. The introduction of new medications also means that the treatment paradigm will change. As urologists treat patients for a longer time with abiraterone and enzalutamide, for example, the patterns of metastases at referral to radiation or medical oncology could be different. Models of care are likely to evolve as well, with the need for multidisciplinary care teams that bridge academic and community interests, perhaps with specific bone metastasis focus groups. The question of who is likely to own radionuclide therapy in the future remains unclear, although it seems certain that institutions will vary in their approaches. Whether radionuclides are administered by nuclear medicine, radiation oncology, or other specialists, it is important that adequate and appropriate credentialing be available and required for all.

The organization of current radionuclide research was discussed, with a focus on efforts to create cooperative studies. It was noted that the field is driven largely by industry, with less current research under way than might be expected from the original promise of TRT. It was stressed that industry alone cannot address the range of scientific questions that should be answered to move the field forward. One challenge cited was the Radiation Therapy Oncology Group reorganization of research interests into organ and body sites, which eliminated the previous radionuclide subgroup active in the 1990s. The discussion group noted that there was no current overlap in research through the SNMMI, Radiation Therapy Oncology Group, or other national groups and recommended that leaders with an interest in radionuclide therapy become involved in key committees of these cooperative groups. SNMMI or NCI might also choose to lead in new non-industry-driven research through a variety of initiatives and mechanisms.

Many questions remain about $\alpha$-particle therapy in bone metastases, and the discussion group identified several key questions that should be the focus of novel research:

- How can we better assess and use information about differential toxicity between normal and malignant tissues?

- Has the maximum tolerated dose of ${ }^{223} \mathrm{Ra}$ and other $\alpha$ emitters been established? Should researchers look more carefully at myeloablation?

- How can we secure more useful data on the dosimetric, pharmacokinetic, and imaging correlates of $\alpha$-emitter therapy in bone metastases? Are bone metastases heterogeneous? If we understand this heterogeneity and the differential responses it engenders, metastases can be targeted in a more rational way.

- How can we enhance the quality of information provided by imaging and assessment of the circulating microenvironment, which are clearly preferable to biopsy?

The discussion group looked at various approaches to new trials, including use of $\alpha$-particle therapy as an adjuvant in highrisk patients with androgen deprivation therapy or in those with suboptimal response to androgen deprivation therapy. Future trials should assess optimal therapeutic protocols for ${ }^{223} \mathrm{Ra}$ in combination or in sequence with novel hormonal agents, combinations of 
novel agents with immunotherapy (such as the work currently under way with ${ }^{153} \mathrm{Sm}$-EDTMP and prostate-specific antigen-TRICOM [Therion Biologics Corp.] vaccine), targeting of the epithelial compartment with nanoparticle delivery of reagents, induction of additive or synergistic DNA damage with cytotoxic chemotherapy or radionuclide targeting, and alternative targeted therapies in prostate cancer, such as blockade of the PI3K/Akt/mTOR (phosphatidylinositol-3' kinase/protein kinase $\mathrm{B} / \mathrm{mammalian}$ target of rapamycin) pathway in combination with radiation.

Basic and translational researchers will need to continue to explore modulation of the treatment microenvironment, beginning with the bone environment. Important questions center on whether $\alpha$ particles can kill cancer stem cells (when these can be identified) and, if so, what cell dose dynamics are involved. Animal models of bone metastasis heterogeneity were discussed, as well as the potential for rapidly transitioning data from such studies into clinical trials. More studies on mouse models of heterogeneity of tumor response to radionuclide therapy are needed, as are well-defined and replicable quantitative imaging approaches that can transition from translational models to clinical trials. The creation of interdisciplinary working groups, including not only imaging specialists but also cancer biologists and others, was suggested as one approach to advancing imaging technologies and techniques.

\section{Solid Tumors}

Dr. Wolfgang Weber summarized the discussions of the breakout group focusing on solid tumors. After a review of the current status of TRT in solid tumors, the group looked in detail at the immediate challenges, the most promising technologic and radiopharmaceutical advances, and the most likely near-term disease targets.

Although targeted radiotherapy has been used clinically in thyroid cancer since the 1940s (4), recent advances in molecular biology have identified a variety of novel targets. Significant progress has been made in the development of ligands binding to these targets, and techniques to calculate and personalize radiation doses.

In current clinical practice, radioiodine therapy is effective as an adjuvant to radioablation in thyroid cancer, treatment for metastatic disease, and modulation of iodine metabolism by protein kinase inhibitors to improve the effectiveness of therapy. Radioiodine therapy may also be enhanced by PET-based radiation dosimetry to predict tumor response (22). Results include improvement in progression-free survival, overall survival, and symptom palliation. Current clinical indications for TRT go beyond thyroid cancer to include ${ }^{90} \mathrm{Y}$ microsphere treatment in liver metastases, for which improvement in progression-free survival has been documented. The discussion group cited several promising preclinical and initial clinical studies that suggest future routine use of TRT in intracavitary therapy of tumors in the central nervous system, intraperitoneal therapy of ovarian cancer, targeting of HER2-expressing tumors, and systemic therapy of prostate cancer (108-110). Two promising example studies described were imaging and therapy of prostate cancer with gastrin-releasing peptide receptor (bombesin) antagonist ${ }^{177} \mathrm{Lu}-\mathrm{RM} 2$ (4-amino-1-carboxymethyl-piperidine-DPhe-Gln-Trp-Ala-Val-Gly-His-Sta-Leu- $\mathrm{NH}_{2}$ ) and case reports of the efficacy of a ${ }^{64} \mathrm{Cu}$-labeled bombesin antagonist $(111,112)$.

The strengths of TRT identified by the discussion group included that, in principle, it can be applied to all malignant tumors; that targets can include tumor cells, tumor stroma, the vasculature, and the physiologic state of the tumor (e.g., pH or hypoxia); and, perhaps most significant, that TRT can be tailored to address the heterogeneity of cancer cells. The most significant weakness of TRT is that specific tumor uptake of currently available radiopharmaceuticals may be suboptimal in some patients.

The issue of heterogeneity was discussed in some detail. Interpatient heterogeneity is well documented, with breast cancers having an average of 60 mutations per tumor compared with 848 mutations per tumor in lung cancer, and 78,775 mutations per tumor in malignant melanoma (113-115). This results in heterogeneity between tumors and can be seen as an important factor in how tumors spread, probably explaining the low response rates in unselected patient populations and rapid development of resistance after initial response. An example of intrapatient heterogeneity in renal cell cancer was detailed (116).

One consensus arising from the group discussion was that TRT is a viable and promising solution for treating heterogeneous tumors. Pretherapeutic imaging in TRT practice can confirm target expression in individual patients and lesions, determine the magnitude of uptake of the therapeutic radiopharmaceutical, estimate radiation dose, and predict treatment efficacy. Tumors not expressing the therapeutic target can be treated by the crossfire effect, with limited resistance to high doses of ionizing radiation. In principle, TRT can address these issues that arise from the heterogeneity of cancer cells and their genetic instability.

Despite the potential for broad efficacy in solid tumors, TRT faces several challenges. These include but are not limited to the fact that the procedure is perceived as complex; requires interdisciplinary collaboration and team science across diverse disciplines; is costly, with significant reimbursement concerns; and is supported by clinical studies of limited size and quality. The group noted that these concerns will most likely be overcome when targeted radiopharmaceuticals have demonstrated clinical benefit in diseases with limited treatment options.

The most promising advances now under investigation are likely to be seen with $\alpha$-particle therapies, nanoparticle-based therapies, novel combinations of imaging and therapy (including radioactive and optical imaging probes developed in tandem with therapeutic radiopharmaceuticals), and combinations of external-beam radiotherapy and TRT. The most promising disease targets under current investigation include prostate cancer (many potential targets), ovarian cancer (intraperitoneal therapy), breast cancer (HER2-directed therapy), brain tumors (intrathecal, intracavitary therapy), and head and neck cancers (p16-negative tumors).

To advance TRT in solid tumors, several scientific and organizational steps are needed. More quantitative analyses of target expression in human tissues are needed to quickly and definitively identify promising targets for radiopharmaceutical development. An infrastructure that supports new radiopharmaceutical development and optimization, and robust, clinically feasible dosimetry, should be encouraged. Interdisciplinary collaborations that drive multiinstitutional trials should be formed, and TRT stakeholders should consider formation of a radiopharmaceutical therapy cooperative trials group, either under the umbrella of an existing organization or as a new effort.

\section{NETs}

Dr. Ananth Srinivasan reported on the breakout group discussion regarding NET therapies. The group looked at both the strengths and the weaknesses of radionuclide therapy in NETs, including the discrepancy between the wide availability of such treatment in Europe and its lack of coverage in the United States, although the possibility of treating patients with NET using ${ }^{177} \mathrm{Lu}$-DOTA-Tyr ${ }^{3}$-octreotate 
(Lutathera; Advanced Accelerator Applications) in the United States through an open trial was mentioned. As in the other breakout sessions, participants called for more basic scientific work to enhance our understanding of the biology of radionuclide therapy.

The group identified the currently most widely used radiopharmaceuticals for therapy in NETs as being ${ }^{90}$ Y-DOTA-Tyr ${ }^{3}$-octreotide (or ${ }^{90}$ Y-DOTATOC or ${ }^{90}$ Y-octreotide), ${ }^{177} \mathrm{Lu}$-DOTA-Tyr ${ }^{3}$-octreotate (or ${ }^{177}$ Lu-DOTATATE or ${ }^{177} \mathrm{Lu}$-octreotate), ${ }^{111}$ In-DTPA-octreotide, ${ }^{131}$ I-MIBG and companion imaging agents for patient selection and following progress (in only a few patients), and ${ }^{68} \mathrm{Ga}-\mathrm{DOTA}-$ octreotide (for PET imaging). ${ }^{111}$ In-DTPA-octreotide is the only FDA-approved imaging agent in use. Other compounds under investigation include ${ }^{90}$ Y-DOTA-Tyr ${ }^{3}$-octreotate and ${ }^{177} \mathrm{Lu}$-DOTA$\mathrm{Tyr}^{3}$-octreotide. Studies performed in Europe over the past $15 \mathrm{y}$ suggest that ${ }^{90} \mathrm{Y}$ - or ${ }^{177} \mathrm{Lu}$-labeled peptides can deliver consistent radiation doses to lesions and achieve significant tumor responses (117).

Current clinical practice with ${ }^{177} \mathrm{Lu}$-DOTA-Tyr ${ }^{3}$-octreotate was reviewed, including details of a decade-long study involving almost 400 patients and representing state-of-the-art practice (118). Results showed that, compared with historical controls, patients undergoing TRT had survival benefits of 40-72 mo, with median time to progression of 40 mo and limited toxicities. Phase III trials have been initiated in the United States and Europe, and the radiopharmaceutical has received orphan drug status.

In a state-of-the-art clinical study with ${ }^{90}$ Y-DOTA-Tyr ${ }^{3}$-octreotide, survival was nearly 3 times longer than expected in patients treated with conventional approaches (117). Median survival from diagnosis was $94.6 \mathrm{mo}, 2.9$ times longer than the expected survival in patients with differentiated metastasized neuroendocrine cancer receiving other treatments (119). However, $12.8 \%$ of patients developed severe transient grade 3-4 hematologic toxicities, with 9.2\% experiencing severe permanent renal toxicity.

Combination TRT is also a current state-of the-art approach in NET treatment. The group cited the example of a study comparing ${ }^{90}$ Y-DOTA-Tyr ${ }^{3}$-octreotide with and without ${ }^{177} \mathrm{Lu}$-DOTA-Tyr ${ }^{3}$ octreotide in almost 500 patients (120). Patients receiving combination therapy had a significantly longer survival than patients receiving ${ }^{90}$ Y-DOTA-Tyr ${ }^{3}$-octreotide alone, with comparable rates of severe hematologic and renal toxicities.

Several ongoing clinical trials of somatostatin receptor-based therapies were reviewed, as was the most recent consensus report of the NCI Neuroendocrine Tumor Clinical Trials Planning Group on future strategies (121). Although key recommendations included creation of randomized phase III studies comparing peptide receptor radiotherapy with standard systemic therapy, the NCI group did not directly address TRT except for the following statement: ". . .many reported studies have had suboptimal methodology, lacked intent-to-treat analyses, and used nonstandard end point definitions. Furthermore, no studies have compared the relative efficacy and toxicity of ${ }^{177}$ Lu-DOTA-Tyr ${ }^{3}$-octreotate and ${ }^{90}$ Y-DOTA-Tyr ${ }^{3}$-octreotide."

Current clinical practice with TRT in NETs is severely limited in the United States, and U.S. patients who are deemed appropriate for TRT (and who can afford the journey) have often gone to specialized European centers, such as those in Rotterdam, The Netherlands; Basel, Switzerland; and Milan, Italy. A small number of patients in the United States are enrolled in other treatment protocols, which in a few centers include ${ }^{131}$ I-MIBG. There is an urgent need to improve access to TRT for NETs in the United States.
The workshop group identified several strengths for TRT in the treatment of NETs. These include the fact that even though a variety of NETs are treated, they all share expression of somatostatin receptor subtype 2 , which can be treated with agents that have already shown efficacy, particularly in median survival and quality of life. Weaknesses include a lack of evidence on complete response rates and well-designed multicenter trials. Additional studies are needed to define the point at which TRT should be initiated, including consideration of TRT as first-line therapy.

Several logistic challenges face widespread adoption of TRT in clinical use. Currently available agents are not approved or reimbursed, with some patents expiring in the short term, making industry less likely to sponsor new research. Cost is an issue for patients and physicians; for example, ${ }^{131} \mathrm{I}-\mathrm{MIBG}$ is reimbursed in some cases but not in others, an area in which SNMMI advocacy might play a role. In many institutions, nuclear medicine physicians are willing to treat patients but patients are not referred, a situation that calls for more collaboration and outreach to endocrinologists and gastrointestinal oncologists. Evidence of treatment efficacy could be accelerated by more methodologies identifying potential responders.

Promising potential advances in somatostatin receptor imaging with antagonists have been shown using radioligands. These radioligands have been shown to label a higher number of receptorbinding sites than conventional agonist radioligands (107). Peptide antagonists labeled with $\beta$ emitters may prove to be useful in combined imaging and therapy (122).

The group addressed areas requiring additional research to advance the field. These include preclinical therapy studies using ${ }^{90} \mathrm{Y} /{ }^{177} \mathrm{Lu}$ antagonists followed by proof-of-concept studies in humans. Human studies with somatostatin receptor antagonists can validate the concept for other indications in which antagonists have been used for imaging. Additional basic studies are needed to better understand radiobiology and develop new dosimetry models. Renal toxicities remain a challenge, and new methods of protecting the kidneys should be explored. Preclinical studies with uptake enhancers to increase receptor expression are also needed.

To accelerate these strategies, the group pointed to the creation of new funding mechanisms that might establish centers of excellence in TRT in different geographic regions of the United States. This not only would provide institutional settings for basic, preclinical, and clinical studies but also would make beneficial treatments more easily available to patients.

\section{WORKSHOP SUMMARY}

Dr. Hossein Jadvar provided an overview and highlights of the workshop, emphasizing areas of consensus, including the regulatory and economic environment, basic biology, and radiochemistry. $\mathrm{He}$ provided a list of current and future issues for consideration, based on presentations at the workshop. Specifically, research is under way, he mentioned, to determine optimal biologic targets and create in vitro and in vivo models to validate new agents and propel them into human trials. High-quality, reproducible data are needed, with basic and preclinical studies performed under good laboratory practices. First-in-human data, including data on absorption, distribution, metabolism, and excretion, will follow, providing information on biologic efficacy, toxicities, and side effects. Among considerations for development and production are ease of radiochemical incorporation (particularly in the generation of theranostic pairs); evaluation of data on specific activity, synthesis yields, chemical and biologic stability, personalized dosimetry, radiobiologic modeling, 
and radiolysis; assessment of the availability of similar or less costly agents with the same action; and easily accessible protocols. Also of importance are the costs of raw materials and the development of reliable distribution networks. Responding to these considerations is not a trivial endeavor.

Few molecular imaging and contrast agents in development make the transition to marketing, and even fewer TRT agents have passed these hurdles in the United States or worldwide. This is in part the result of the economic and regulatory landscape in which these agents are developed, approved, and marketed. Market size, market share, price resistance, and likelihood of reimbursement affect industry decisions on whether and how to develop a new agent, even when that agent is clearly beneficial. Challenges include the cost of supporting clinical trials needed for approval, issues of exclusivity in terms of patent protection, market competition from same-disease-targeted agents, and a regulatory environment that places constraints on dosimetry, administration, transport, and waste disposal.

A major challenge for TRT is ready availability of radioisotopes for research and clinical use. Some radioisotopes are not available in the United States; others are unavailable for clinical use because of lack of reimbursement and approval; some are available but underused in the clinic. Integration and determination of optimal sequencing of TRT with existing standards of care might advance clinical use, as would better data on side effects, toxicity, and complications compared with conventional therapies. More attention to patient-reported outcomes assessing quality-of-life benefits with TRT is also needed.

Although data on TRT efficacy are available from other countries, non-U.S. data are rarely sufficient for approval and reimbursement in the United States. The result is that for U.S. approval, scientists are often forced to reinvent the wheel, duplicating studies that have already been published in respected peer-reviewed literature. One challenge that should be considered by stakeholders is to identity ways to propel existing data on TRT forward more quickly in support of U.S. approvals. Another challenge is to directly address the various logistic problems cited in the workshop: limited access to TRT for patients and providers, perceived high cost, turf issues, patient perceptions and fears, and the need for multidisciplinary teams supported by strong TRT education efforts (123-132).

During his tenure as director of the National Institutes of Health, Dr. Elias Zerhouni introduced the 4 Ps of medicine: personalized, predictive, preemptive, and participatory. These apply to TRT, with the additional need for partnership. Education and collaboration are key to ensuring a multidisciplinary approach. Stakeholders involved in such collaborations are numerous and include federal agencies, academia, pharmaceutical companies, patients and patient advocacy groups, providers (preferably in multispecialty teams), payers, professional societies, and philanthropists and venture capitalists. The ultimate goal, as in all of medicine, is to ensure that patients receive timely, appropriate therapy in a cost-effective manner. Balancing these forces through multidisciplinary collaboration, bench-to-bedside discovery, innovation, documentation, and validation of enhanced outcomes is a challenge that will require new cooperative mechanisms and the involvement of the broadest range of stakeholders interested in advancing TRT.

\section{CONCLUSION}

TRT has great potential for cancer therapy across a spectrum of tumor types including breast, lung, bone, lymphoma, and NETs, among others, and may be particularly helpful to treat heterogeneous tumors. TRT could be applied as a primary therapy or in conjunction with other approaches, including external-beam radiation or brachytherapy. However, TRT requires a multidisciplinary approach, and for this potential to be realized, cooperative structures must be developed across institutions to gather the necessary basic science and clinical trial data. This research most likely will need to go far beyond that which is typically industry-sponsored. Therefore, a multiinstitutional infrastructure would likely be helpful to facilitate the collection of such data.

As new potential agents are developed, knowledge of the microenvironment and metabolism both in the tumor and in normal tissue must be sought, leading to patient-specific radiobiology-based dosimetric models and allowing for effective treatment planning. Effort should also be made to establish routine production at high activity levels. New, effective models and production methods will also be desirable for agents in current use. Since no single institution will likely have expertise in all areas, a multiinstitutional research network may be effective in enhancing the advancement of this research.

Once an agent is ready for in-human investigations, large-scale randomized, evidence-based clinical trials that establish its effectiveness with respect to cost, quality of life, and patient outcome will be needed. Study design and patient selection are critical for implementation, and time will be of the essence so as to allow comparison to current alternative therapies. This will most likely require a multiinstitutional cooperative infrastructure beyond that provided through industry-based trials.

The clinical implementation of TRT will require a multidisciplinary approach and education of all involved, including basic scientists, physicians, and support personnel. Further careful treatment planning and patient selection will be needed to ensure treatment benefit.

This workshop was unique in that it included a wide range of stakeholders invested in the success of TRT, including a variety of clinicians (nuclear medicine physicians, radiation oncologists, and medical oncologists) and basic scientists (radiochemists, physicists, and radiobiologists). In order for the promise of TRT to be realized, more such gatherings would be helpful since the most successful implementation will be multidisciplinary. Although focus on a particular disease such as lymphoma or bone disease may be helpful, interdisciplinary cooperation will clearly be critical, both across and within institutions.

\section{ACKNOWLEDGMENTS}

The Organizing Committee is made up of the following individuals: Frederic Fahey, DSc (Boston Children's Hospital, Boston, Massachusetts, and Harvard Medical School, Boston, Massachusetts); Katherine Zukotynski, MD, FRCPC (Sunnybrook Health Sciences Centre, University of Toronto, Ontario, Canada, and Brigham and Women's Hospital, Harvard Medical School, Boston, Massachusetts); Jacek Capala, PhD (National Cancer Institute/NIH, Bethesda, Maryland); Hossein Jadvar, MD, PhD, MPH, MBA, FACNM (University of Southern California, Los Angeles, California); Gary Dillehay, MD, FACNM, FACR (Northwestern University Medical School, Chicago, Illinois); Peter Herscovitch, MD (National Institutes of Health Clinical Center, Bethesda, Maryland); Nancy Knight, $\mathrm{PhD}$ (University of Maryland School of Medicine, Baltimore, Maryland); and Virginia Pappas, CAE (SNMMI, Reston, Virginia).

The contributors include George Sgouros, PhD (Johns Hopkins University School of Medicine, Baltimore, Maryland); Michael R. 
Zalutsky, PhD (Duke University Medical Center, Durham, North Carolina); Richard L. Wahl, MD (Johns Hopkins University School of Medicine, Baltimore, Maryland); Eric Jacobsen, MD (DanaFarber Cancer Institute, Boston, Massachusetts, and Harvard Medical School, Boston, Massachusetts); David I. Quinn, MBBS, PhD, FRACP (USC Norris Comprehensive Cancer Center, Keck School of Medicine, University of Southern California, Los Angeles, California); Jorge A. Carrasquillo, MD (Memorial Sloan-Kettering Cancer Center, New York, New York); Bhadrasain Vikram, MD (National Cancer Institute/NIH, Radiation Research Program, Bethesda, Maryland); Steven Larson, MD, FACNM (Memorial Sloan-Kettering Cancer Center, New York, New York); Janis P. O'Malley, MD (University of Alabama at Birmingham, Birmingham, Alabama); Wolfgang Weber, MD (Memorial SloanKettering Cancer Center, New York, New York); and Ananth Srinivasan, PhD (Stanford University, Palo Alto, California).

The participants include Robert R. Bahnson, MD, FACS (Ohio State Wexner Medical Center, Columbus, Ohio); Martin W. Brechbiel, $\mathrm{PhD}$ (National Cancer Institute, Bethesda, Maryland); David Y.T. Chen, MD, FACS (Fox Chase Cancer Center, Rockledge, Pennsylvania); Xiaoyuan Shawn Chen, PhD (National Institute of Biomedical Imaging and Bioengineering, Bethesda, Maryland); Norman Coleman, MD (National Cancer Institute, Rockville, Maryland); Adam P. Dicker, MD, PhD (Jefferson Medical College of Thomas Jefferson University, Philadelphia, Pennsylvania); David M. Goldenberg, ScD, MD (Immunomedics, Inc., Morris Plains, New Jersey); Roger W. Howell, PhD (Rutgers New Jersey Medical School, Newark, New Jersey); Edvardas Kaminskas, MD (Office of Hematology and Oncology Products, Food and Drug Administration, Silver Spring, Maryland); Adam Opanowski, CNMT, PET, NCT, RT(N) (ACR Clinical Research Center Core Laboratory, Philadelphia, Pennsylvania); Karel Pacak, MD (National Institute of Child Health and Human Development, Bethesda, Maryland); Daniel Pryma, MD (University of Pennsylvania, Philadelphia, Pennsylvania); Tod W. Speer, MD (University of Wisconsin School of Medicine and Public Health, Madison, Wisconsin); Suresh C. Srivastava, $\mathrm{PhD}$ (Brookhaven National Laboratory, Upton, New York); Cindy Tomlinson (American Society for Radiation, Fairfax, Virginia); John F. Valliant, PhD (McMaster University, Hamilton, Ontario, Canada); Barry W. Wessels, PhD (University Hospitals-Case Western Reserve University, Cleveland, Ohio); and D. Scott Wilbur, PhD (University of Washington, Seattle, Washington).

The contributions of Terpse Gentile, Nikki Wenzel-Lamb, and Amelia Ballinger (SNMMI) to this work are gratefully acknowledged.

\section{REFERENCES}

1. Joint NCI/SNMMI Workshop on Targeted Radionuclide Therapy. J Nucl Med. 2013;54(5):16N-17N.

2. Sgouros G, Roeske JC, McDevitt MR, et al. MIRD pamphlet no. 22: radiobiology and dosimetry of $\alpha$-particle emitters for targeted radionuclide therapy. http:// interactive.snm.org/docs/MIRD_alpha_paper_Mar\%2011_09_Jun\%2019_09.pdf. Accessed November 19, 2013.

3. Bell AG. The uses of radium. Am Med. 1903;6:261-266.

4. Seidlin SM, Marinelli LD, Oshry E. Radioactive iodine therapy: effect on functioning metastases of adenocarcinoma of the thyroid. J Am Med Assoc. 1946; 132:838-847.

5. Larson SM, Carrasquillo JA, Krohn KA, et al. Localization of ${ }^{131}$ I-labeled p97specific Fab fragments in human melanoma as a basis for radiotherapy. J Clin Invest. $1983 ; 72: 2101-2114$

6. Jurcic JG, McDevitt MR, Sgouros G, et al. Targeted alpha-particle therapy for myeloid leukemias: a phase I trial of bismuth-213-HuM195 (anti-CD33) [abstract]. Blood. 1997;90:2245.
7. Harrison MR, Wong TZ, Armstrong AJ, George DJ. Radium-223 chloride: a potential new treatment for castration-resistant prostate cancer patients with metastatic bone disease. Cancer Manag Res. 2013;5:1-14.

8. Perkins A. In vivo molecular targeted radiotherapy. Biomed Imaging Interv J. 2005; 1:e9.

9. Sgouros G, Barest G, Thekkumthala J, et al. Treatment planning for internal radionuclide therapy: three-dimensional dosimetry for nonuniformly distributed radionuclides. J Nucl Med. 1990;31:1884-1891.

10. Prideaux AR, Song H, Hobbs RF, et al. Three-dimensional radiobiologic dosimetry: application of radiobiologic modeling to patient-specific 3-dimensional imaging-based internal dosimetry. J Nucl Med. 2007;48:1008-1016.

11. Kolbert KS, Sgouros G, Scott AM, et al. Implementation and evaluation of patient-specific three-dimensional internal dosimetry. J Nucl Med. 1997;38:301-308.

12. Baechler S, Hobbs RF, Prideaux AR, Wahl RL, Sgouros G. Extension of the biological effective dose to the MIRD schema and possible implications in radionuclide therapy dosimetry. Med Phys. 2008;35:1123-1134.

13. Barone R, Borson-Chazot F, Valkema R, et al. Patient-specific dosimetry in predicting renal toxicity with ${ }^{90} \mathrm{Y}$-DOTATOC: relevance of kidney volume and dose rate in finding a dose-effect relationship. J Nucl Med. 2005;46(suppl 1): 99S-106S.

14. Hobbs RF, Sgouros G. Calculation of the biological effective dose for piecewise defined dose-rate fits. Med Phys. 2009;36:904-907.

15. Hobbs RF, Wahl RL, Lodge MA, et al. ${ }^{124}$ I-PET-based 3D-RD dosimetry for a pediatric thyroid cancer patient: real-time treatment planning and methodologic comparison. J Nucl Med. 2009;50:1844-1847.

16. Hobbs RF, McNutt $T$, Baechler $S$, et al. A treatment planning method for sequentially combining radiopharmaceutical therapy and external radiation therapy. Int J Radiat Oncol Biol Phys. 2011;80:1256-1262.

17. Watchman CJ, Jokisch DW, Patton PW, Rajon DA, Sgouros G, Bolch WE. Absorbed fractions for alpha-particles in tissues of trabecular bone: considerations of marrow cellularity within the ICRP reference male. $\mathrm{J} \mathrm{Nucl} \mathrm{Med}$. 2005;46:1171-1185.

18. Song H, Shahverdi K, Huso DL, et al. ${ }^{213} \mathrm{Bi}$ (alpha-emitter)-antibody targeting of breast cancer metastases in the neu-N transgenic mouse model. Cancer Res. 2008;68:3873-3880.

19. Mercadante S, Fulfaro F. Management of painful bone metastases. Curr Opin Oncol. 2007;19:308-314.

20. Akabani G, Carlin S, Welsh P, Zalutsky MR. In vitro cytotoxicity of ${ }^{211}$ At-labeled trastuzumab in human breast cancer cell lines: effect of specific activity and HER2 receptor heterogeneity on survival fraction. Nucl Med Biol. 2006;33:333-347.

21. Schlumberger M, Catargi B, Borget I, et al. Strategies of radioiodine ablation in patients with low-risk thyroid cancer. N Engl J Med. 2012;366:1663-1673.

22. Ho AL, Grewal RK, Leboeuf R, et al. Selumetinib-enhanced radioiodine uptake in advanced thyroid cancer. N Engl J Med. 2013;368:623-632.

23. Seldin DW. Techniques for using Bexxar for the treatment of non-Hodgkin's lymphoma. J Nucl Med Technol. 2002;30:109-114.

24. Stashenko P, Nadler LM, Hardy R, Schlossman SF. Characterization of a human B lymphocyte-specific antigen. J Immunol. 1980;125:1678-1685.

25. Kaminski MS, Radford JA, Gregory SA, et al. Re-treatment with I-131 tositumomab in patients with non-Hodgkin's lymphoma who had previously responded to I-131 tositumomab. J Clin Oncol. 2005;23:7985-7993.

26. Kaminski MS, Tuck M, Estes J, et al. ${ }^{131}$ I-tositumomab therapy as initial treatment for follicular lymphoma. N Engl J Med. 2005;352:441-449.

27. Leahy MF, Seymour JF, Hicks RJ, Turner JH. Multicenter phase II clinical study of iodine-131-rituximab radioimmunotherapy in relapsed or refractory indolent non-Hodgkin's lymphoma. J Clin Oncol. 2006;24:4418-4425.

28. Press OW, Unger JM, Braziel RM, et al. Phase II trial of CHOP chemotherapy followed by tositumomab/iodine I-131 tositumomab for previously untreated follicular non-Hodgkin's lymphoma: five-year follow-up of Southwest Oncology Group Protocol S9911. J Clin Oncol. 2006;24:4143-4149.

29. Witzig TE, Gordon LI, Cabanillas F, et al. Randomized controlled trial of yttrium-90-labeled ibritumomab tiuxetan radioimmunotherapy versus rituximab immunotherapy for patients with relapsed or refractory low-grade, follicular, or transformed B-cell non-Hodgkin's lymphoma. J Clin Oncol. 2002;20:2453-2463.

30. Morschhauser F, Radford J, Van Hoof A, et al. ${ }^{90}$ Yttrium-ibritumomab tiuxetan consolidation of first remission in advanced-stage follicular non-Hodgkin lymphoma: updated results after a median follow-up of 7.3 years from the international, randomized, phase III first-line indolent trial. J Clin Oncol. 2013;31:1977-1983.

31. He B, Wahl RL, Sgouros G, et al. Comparison of organ residence time estimation methods for radioimmunotherapy dosimetry and treatment planning: patient studies. Med Phys. 2009;36:1595-1601.

32. Kwekkeboom DJ, de Herder WW, Krenning EP. Somatostatin receptor-targeted radionuclide therapy in patients with gastroenteropancreatic neuroendocrine tumors. Endocrinol Metab Clin North Am. 2011;40:173-185. 
33. Matthay KK, Tan JC, Villablanca JG, et al. Phase I dose escalation of iodine131-metaiodobenzylguanidine with myeloablative chemotherapy and autologous stem-cell transplantation in refractory neuroblastoma: a New Approaches to Neuroblastoma Therapy Consortium Study. J Clin Oncol. 2006;24:500-506.

34. Matthay KK, Weiss B, Villablanca JG, et al. Dose escalation study of no-carrieradded ${ }^{131}$ I-metaiodobenzylguanidine for relapsed or refractory neuroblastoma: New Approaches to Neuroblastoma Therapy Consortium Trial. J Nucl Med. 2012;53: 1155-1163.

35. Schaefer NG, Ma J, Huang P, Buchanan J, Wahl RL. Radioimmunotherapy in non-Hodgkin lymphoma: opinions of U.S. medical oncologists and hematologists. J Nucl Med. 2010;51:987-994.

36. Rummel MJ, Niederle N, Maschmeyer G, et al. Bendamustine plus rituximab versus CHOP plus rituximab as first-line treatment for patients with indolent and mantle cell lymphomas: an open-label, multicenter, randomized, phase 3 noninferiority trial. Lancet. 2013;381:1203-1210.

37. Martinelli G, Schmitz SF, Utiger U, et al. Long-term follow-up of patients with follicular lymphoma receiving single-agent rituximab at 2 different schedules in trial SAKK 37/98. J Clin Oncol. 2010;28:4480-4484.

38. Salles G, Seymour JF, Offner F, et al. Rituximab maintenance for 2 years in patients with high tumour burden follicular lymphoma responding to rituximab plus chemotherapy (PRIMA): a phase 3, randomised controlled trial. Lancet. 2011;377:42-51.

39. Kaminski MS, Tuck M, Estes J, et al. Tositumomab and iodine I-131 tositumomab for previously untreated, advanced-stage, follicular lymphoma: median 10 year follow-up results. Paper presented at: 51st ASH Annual Meeting and Exposition, December 5-8, 2009, New Orleans, LA. https://ash. confex.com/ash/2009/webprogram/Paper24876.html. Updated December 3, 2009. Accessed November 19, 2013.

40. Ardeshna KM, Qian W, Smith P, et al. An intergroup randomized trial of rituximab versus a watch and wait strategy in patients with stage II, II, IV, asymptomatic, non-bulky follicular lymphoma (grades 1,2 and 3a): a preliminary analysis [abstract]. Blood. 2010; 115:6.

41. Ardeshna KM, Smith P, Norton A, et al. Long-term effect of a watch and wait policy versus immediate treatment for asymptomatic advanced-stage nonHodgkin lymphoma: a randomized controlled trial. Lancet. 2003;362:516-522.

42. Kahl BS, Bartlett NL, Leonard JP, et al. Bendamustine is effective therapy in patients with rituximab-refractory indolent B-cell non-Hodgkin lymphoma: results from a multicenter study. Cancer. 2010;116:106-114.

43. Press OW, Unger JM, Rimza L, et al. Phase III randomized intergroup trial of CHOP plus rituximab compared with CHOP chemotherapy plus ${ }^{131}$ iodine-tositumomab for previously untreated follicular non-Hodgkin lymphoma: SWOG S0016. J Clin Oncol. 2013;31:314-320.

44. Morschhauser F, Radford J, Van Hoof A, et al. Phase III trial of consolidation therapy with yttrium-90-ibritumomab tiuxetan compared with no additional therapy after first remission in advanced follicular lymphoma. J Clin Oncol. 2008;26:5156-5164.

45. Morschhauser F, Illidge T, Huglo D, et al. Efficacy and safety of yttrium-90 ibritumomab tiuxetan in patients with relapsed or refractory diffuse large B-cell lymphoma not appropriate for autologous stem-cell transplantation. Blood. 2007;110:54-58.

46. Hamlin PA, Rodriguez MA, Nov A, et al. Final results of a phase II study of sequential R-CHOP and yttrim-90 ibritumomab tiuxetan (RIT) for elderly high risk patients with untreated diffuse large B-cell lymphoma (DLBCL) [abstract]. Blood. 2010;116:abstract 1793.

47. Vose JM, Carter S, Burns LJ, et al. Phase III randomized study of rituximab/ carmustine, etoposide, cytarabine, and melphalan (BEAM) compared with iodine-131 tositumomab/BEAM with autologous hematopoietic cell transplantation for relapsed diffuse large B-cell lymphoma: results from the BMY CTN 0401 trial. J Clin Oncol. 2013;31:1662-1668.

48. Smith MRLH, Gordon L, Gascoyne RD, et al. Phase II study of rituximab plus cyclophosphamide, doxorubicin, vincristine, and prednisone immunochemotherapy followed by yttrium-90-ibritumomab tiuxetan in untreated mantle cell lymphoma: Eastern Cooperative Oncology Group Study E1499. J Clin Oncol. 2012;30:3119-3126.

49. Kluin-Nelemans HC, Hoster E, Hermine O, et al. Treatment of older patients with mantle-cell lymphoma. N Engl J Med. 2012;367:520-531.

50. Crawford ED, Eisenberger MA, McLeod DG, et al. A controlled trial of leuprolide with and without flutamide in prostatic carcinoma. N Engl J Med. 1989;321:419-424.

51. Tannock IF, de Wit R, Berry WR, et al. Docetaxel plus prednisone or mitoxantrone plus prednisone for advanced prostate cancer. N Engl J Med. 2004;351:1502-1512.

52. Tannock IF, Osoba D, Stockler MR, et al. Chemotherapy with mitoxantrone plus prednisone or prednisone alone for symptomatic hormone-resistant prostate cancer: a Canadian randomized trial with palliative end points. J Clin Oncol. 1996;14:1756-1764
53. The Leuprolide Study Group. Leuprolide versus diethylstilbestrol for metastatic prostate cancer. N Engl J Med. 1984;311:1281-1286.

54. Saad F, Gleason DM, Murray R, et al. A randomized, placebo-controlled trial of zoledronic acid in patients with hormone-refractory metastatic prostate carcinoma. J Natl Cancer Inst. 2002;94:1458-1468.

55. Petrylak DP, Tangen CM, Hussain MHA, et al. Docetaxel and estramustine compared with mitoxantrone and prednisone for advanced refractory prostate cancer. N Engl J Med. 2004;351:1513-1520.

56. de Bono JS, Logothetis CJ, Molina A, et al. Abiraterone and increased survival in metastatic prostate cancer. N Engl J Med. 2011;364:1995-2005.

57. Fizazi K, Carducci M, Smith M, et al. Denosumab versus zoledronic acid for treatment of bone metastases in men with castration-resistant prostate cancer: a randomized, double-blind study. Lancet. 2011;377:813-822.

58. Scher HI, Fizazi K, Saad F, et al. Increased survival with enzalutamide in prostate cancer after chemotherapy. N Engl J Med. 2012;367:1187-1197.

59. Kantoff PW, Higano CS, Shore ND, et al. Sipuleucel-T immunotherapy for castration-resistant prostate cancer. $N$ Engl J Med. 2010;363:411-422.

60. Lipton A, Small E, Saad F, et al. The new bisphosphonate, Zometa (zoledronic acid), decreases skeletal complications in both osteolytic and osteoblastic lesions: a comparison to pamidronate. Cancer Invest. 2002;20(suppl 2):45-54.

61. Tu SM, Millikan RE, Mengistu B, et al. Bone-targeted therapy for advanced androgen-dependent carcinoma of the prostate: a randomized phase II trial. Lancet. 2001;357:336-341.

62. Parker C, Nilsson S, Heinrich, et al. Alpha emitter radium-223 and survival in metastatic prostate cancer. N Engl J Med. 2013;369:213-223.

63. Bruland ØS, Nilsson S, Fisher DR, Larsen RH. High-linear energy transfer irradiation targeted to skeletal metastases by the alpha-emitter ${ }^{223} \mathrm{Ra}$ : adjuvant or alternative to conventional modalities? Clin Cancer Res. 2006;12(suppl):6250s-6257s.

64. Kassis AI. Therapeutic radionuclides: biophysical and radiobiologic principles. Semin Nucl Med. 2008;38:358-366.

65. Wieland DM, Wu J, Brown LE, Mangner TJ, Swanson DP, Beierwaltes WH. Radiolabeled adrenergic neuron-blocking agents: adrenomedullary imaging with ${ }^{131}$ I-metaiodobenzylguanidine. J Nucl Med. 1980;21:349-353.

66. Shapiro B, Sisson JC, Wieland DM, et al. Radiopharmaceutical therapy of malignant pheochromocytoma with $\left[{ }^{131} \mathrm{I}\right]$ metaiodobenzylguanidine: results from 10 years experience. J Nucl Biol Med. 1991;35:269-276.

67. Fischer M. Therapy of pheochromocytoma with ${ }^{131}$ I-metaiodobenzylguanidine. J Nucl Biol Med. 1991;35:292-294.

68. Lumbroso J, Schlumberger M, Tenenbaum F, Aubert B, Travagli JP, Parmentier C. ${ }^{131}$ I-metaiodobenzylguanidine therapy in 20 patients with malignant pheochromocytoma. J Nucl Biol Med. 1991;35:288-291.

69. Schlumberger M, Gicquel C, Lumbroso J, et al. Malignant pheochromocytoma: clinical, biological, histologic, and therapeutic data in a series of 20 patients with distant metastases. J Endocrinol Invest. 1992;15:631-642.

70. Bestagno M, Pizzocaro C, Maira G, Terzi A, Panarotto MB, Guerra P. Results of $\left[{ }^{131} \mathrm{I}\right]$ metaiodobenzylguanidine treatment in metastatic malignant phaeochromocytoma. J Nucl Biol Med. 1991;35:277-279.

71. Krempf M, Lumbroso J, Mornex R, et al. Treatment of malignant pheochromocytoma with ${ }^{131}$ I-metaiodobenzylguanidine: a French multicenter study. J Nucl Biol Med. 1991;35:284-287.

72. Krempf M, Lumbroso J, Mornex R, et al. Use of $\mathrm{m}^{-{ }^{131}}$ I-iodobenzylguanidine in the treatment of malignant pheochromocytoma. J Clin Endocrinol Metab. 1991;72:455-461.

73. Shilkrut M, Bar-Deroma R, Bar-Sela G, Berniger A, Kuten A. Low-dose iodine131 metaiodobenzylguanidine therapy for patients with pheochromocytoma and paraganglioma: single center experience. Ann J Clin Oncol. 2010;33:79-82.

74. Sisson JC, Shapiro B, Beierwaltes WH, Copp JE. Locating pheochromocytomas by scintigraphy using ${ }^{131}$ I-metaiodiobenzylguanidine. CA Cancer J Clin. 1984;34: 86-92.

75. Sisson JC, Shapiro B, Beierwaltes WH, et al. Radiopharmaceutical treatment of malignant pheochromocytoma. J Nucl Med. 1984;25:197-206.

76. Sisson JC, Shapiro B, Beierwaltes WH. Scintigraphy with ${ }^{131}$ I-MIBG as an aid to the treatment of pheochromocytomas in patients with multiple endocrine neoplasia type 2 syndromes. Henry Ford Hosp Med J. 1984;32:254-261.

77. Loh KC, Fitzgerald PA, Matthay KK, Yeo PP, Price DC. The treatment of malignant pheochromocytoma with iodine-131 metaiodobenzylguanidine ( $\left.{ }^{131} \mathrm{I}-\mathrm{MIBG}\right)$ : a comprehensive review of 116 reported patients. J Endocrinol Invest. 1997;20: 648-658.

78. Mukherjee JJ, Kaltsas GA, Islam N, et al. Treatment of metastatic carcinoid tumours, phaeochromocytoma, paraganglioma and medullary carcinoma of the thyroid with ${ }^{131}$ I-meta-iodobenzylguanidine. Clin Endocrinol (Oxf). 2001;55:46-60.

79. Castellani MR, Seghezzi S, Chiesa C, et al. ${ }^{131} \mathrm{I}-\mathrm{MIBG}$ treatment of pheochromocytoma: low versus intermediate activity regimens of therapy. Q J Nucl Med Mol Imaging. 2010;54:100-113. 
80. Hartley A, Spooner D, Brunt AM. Management of malignant phaeochromocytoma: a retrospective review of the use of MIBG and chemotherapy in the West Midlands. Clin Oncol (R Coll Radiol). 2001;13:361-366.

81. Gedik GK, Hoefnagel CA, Bais E, Olmos Ra ${ }^{131} \mathrm{I}-\mathrm{MIBG}$ therapy in metastatic phaeochromocytoma and paraganglioma. Eur J Nucl Med Mol Imaging. 2008; 35:725-733.

82. Safford SD, Coleman RE, Gockerman JP, et al. Iodine-131 metaiodiobenzylguanidine is an effective treatment for malignant pheochromocytoma and paraganglioma. Surgery. 2003;134:956-962.

83. Bomanji JB, Wong W, Gaze MN, et al. Treatment of neuroendocrine tumours in adults with ${ }^{131}$ I-MIBG therapy. Clin Oncol (R Coll Radiol). 2003;15:193-198.

84. Rose B, Matthay KK, Price D, et al. High-dose ${ }^{131}$ I-metaiodobenzylguanidine therapy for 12 patients with malignant pheochromocytoma. Cancer. 2003;98: 239-248.

85. Gonias S, Goldsby R, Matthay KK, et al. Phase II study of high-dose ${ }^{131} \mathrm{I}-$ metaiodobenzylguanidine therapy for patients with metastatic pheochromocytoma and paraganglioma. J Clin Oncol. 2009;27:4162-4168.

86. Reubi JC, Schar JC, Waser B, et al. Affinity profiles for human somatostatin receptor subtypes SST1-SST5 of somatostatin radiotracers selected for scintigraphic and radiotherapeutic use. Eur J Nucl Med. 2000;27:273-282.

87. Breeman WA, De Jong M, Visser TJ, Erion JL, Krenning EP. Optimising conditions for radiolabelling of DOTA-peptides with ${ }^{90} \mathrm{Y},{ }^{111} \mathrm{In}$, and ${ }^{177} \mathrm{Lu}$ at high specific activities. Eur J Nucl Med Mol Imaging. 2003;30:917-920.

88. Valkema R, Pauwels S, Kvols LK, et al. Survival and response after peptide receptor radionuclide therapy with [ ${ }^{90}$ Y-DOTA0,Tyr3]octreotide in patients with advanced gastroenteropancreatic neuroendocrine tumors. Semin Nucl Med. 2006; 36:147-156.

89. Nguyen C, Faraggi M, Giraudet AL, et al. Long-term efficacy of radionuclide therapy with disseminated neuroendocrine tumors uncontrolled by conventional therapy. J Nucl Med. 2004;45:1660-1668.

90. Paganelli G, Bodei L, Handkiewicz Junak D, et al. ${ }^{90}$ Y-DOTA-D-Phe1-Try3octreotide in therapy of neuroendocrine malignancies. Biopolymers. 2002;66: 393-398.

91. de Jong M, Breeman WA, Kwekkeboom DJ, Valkema R, Krenning EP. Tumor imaging and therapy using radiolabeled somatostatin analogues. Acc Chem Res. 2009; 42:873-880

92. van Essen M, Krenning EP, Kam BL, de Herder WW, Feelders RA, Kwekkeboom DJ. Salvage therapy with ${ }^{177} \mathrm{Lu}$-octreotate in patients with bronchial and gastroenteropancreatic neuroendocrine tumors. J Nucl Med. 2010;51:383-390.

93. Waldherr C, Pless M, Maecke HR, et al. Tumor response and clinical benefit in neuroendocrine tumors after $7.4 \mathrm{GBq}{ }^{90}$ Y-DOTATOC. J Nucl Med. 2002;43: 610-616.

94. Waldherr C, Pless M, Maecke HR, Haldemann A, Mueller-Brand J. The clinical value of ${ }^{90}$ Y-DOTA-D-Phe1-Tyr3-octreotide ( ${ }^{\circ}$ Y-DOTATOC) in the treatment of neuroendocrine tumours: a clinical phase II study. Ann Oncol. 2001;12:941-945.

95. Forrer F, Riedweg I, Maecke HR, Mueller-Brand J. Radiolabeled DOTATOC in patients with advanced paraganglioma and pheochromocytoma. $Q \mathrm{~J} \mathrm{Nucl} \mathrm{Med}$ Mol Imaging. 2008;52:334-340.

96. Forrer F, Uusijarvi H, Storch D, Maecke HR, Mueller-Brand J. Treatment with ${ }^{177} \mathrm{Lu}$-DOTATOC of patients with relapse of neuroendocrine tumors after treatment with ${ }^{90}$ Y-DOTATOC. $J$ Nucl Med. 2005;46:1310-1316.

97. Otte A, Herrmann R, Heppeler A, et al. Yttrium-90 DOTATOC: first clinical results. Eur J Nucl Med. 1999;26:1439-1447.

98. Buchmann I, Henze M, Engelbrecht $\mathrm{S}$, et al. Comparison of ${ }^{68} \mathrm{Ga}-\mathrm{DOTATOC}$ PET and ${ }^{111}$ In-DTPAOC (Octreoscan) SPECT in patients with neuroendocrine tumours. Eur J Nucl Med Mol Imaging. 2007;34:1617-1626.

99. Hartmann H, Zophel K, Freudenberg R, et al. Radiation exposure of patients during ${ }^{68} \mathrm{Ga}$-DOTATOC PET/CT examinations [in German]. Nuklearmedizin. 2009;48:201-207.

100. Pfeifer A, Knigge U, Mortensen J, et al. Clinical PET of neuroendocrine tumors using ${ }^{64} \mathrm{Cu}$-DOTATATE: first-in-humans study. J Nucl Med. 2012;53:1207-1215.

101. Jamar F, Barone R, Mathieu I, et al. ${ }^{86}$ Y-DOTA0)-D-Phe1-Tyr3-octreotide (SMT487): a phase 1 clinical study-pharmacokinetics, biodistribution and renal protective effect of different regimens of amino acid co-infusion. Eur J Nucl Med Mol Imaging. 2003;30:510-518.

102. Kratochwil C, Giesel FL, Lopez-Benitez R, et al. Intraindividual comparison of selective arterial versus venous ${ }^{68} \mathrm{Ga}$-DOTATOC PET/CT in patients with gastroenteropancreatic neuroendocrine tumors. Clin Cancer Res. 2010;16:2899_ 2905.

103. Kratochwil C, Lopez-Benitez R, Mier W, et al. Hepatic arterial infusion enhances DOTATOC radiopeptide therapy in patient with neuroendocrine liver metastases. Endocr Relat Cancer. 2011;18:595-602.
104. Bass RT, Buckwalter BL, Patek BP, et al. Identification and characterization of novel somatostatin antagonists. Mol Pharmacol. 1996;50:709-715.

105. Cescato R, Erchegyi K, Waser B, et al. Design and in vitro characterization of highly sst2-selective somatostatin antagonists suitable for radiotherapy. $J$ Med Chem. 2008;51:4030-4037.

106. Cescato R, Waser B, Fani M, Reubi JC. Evaluation of ${ }^{177}$ Lu-DOTA-sst2 antagonist versus ${ }^{177} \mathrm{Lu}$-DOTA-sst 2 agonist binding in human cancers in vitro. $\mathrm{J} \mathrm{Nucl}$ Med. 2011;52:1886-1890.

107. Wild D, Fani M, Behe M, et al. First clinical evidence that imaging with somatostatin receptor antagonists is feasible. J Nucl Med. 2011;52:1412-1417.

108. Kneifel S, Cordier D, Good S, et al. Local targeting of malignant gliomas by the diffusible peptidic vector, 1,4,7,10-tetraazacyclododecane-1-glutaric acid4,7,10-triacetic acid-substance p. Clin Cancer Res. 2006;12:3843-3850.

109. Kramer K, Humm JL, Souweidane MM, et al. Phase I study of targeted radioimmunotherapy for leptomeningeal cancers using intra-Ommaya ${ }^{131} \mathrm{I}-3 \mathrm{~F} 8$. J Clin Oncol. 2007;25:5465-5470.

110. Song H, Hobbs RF, Vajravelu R, et al. Radioimmunotherapy of breast cancer metastases with alpha-particle emitter ${ }^{225} \mathrm{Ac}$ : comparing efficacy with ${ }^{213} \mathrm{Bi}$ and ${ }^{90}$ Y. Cancer Res. 2009;69:8941-8948.

111. Dumont RA, Tamma M, Braun F, et al. Targeted radiotherapy of prostate cancer with a gastrin-releasing peptide receptor antagonist is effective as a monotherapy and in combination with rapamycin. J Nucl Med. 2013;54:762-769.

112. Gornik G, Mansi R, Abiraj K, et al. Evaluation of the GRPR radioantagonist $\mathrm{Cu}-$ 64-CB-TE2A-AR-06 in mice and men [abstract]. J Nucl Med. 2011;52(suppl):7P

113. Cancer Genome Atlas Network. Comprehensive molecular characterization of human colon and rectal cancer. Nature. 2012;487:330-337.

114. Cancer Genome Atlas Network. Comprehensive molecular portraits of human breast tumours. Nature. 2012;490:61-70.

115. Cancer Genome Atlas Research Network. Comprehensive genomic characterization of squamous cell lung cancers. Nature. 2012;489:519-525.

116. Gerlinger M, Rowan AJ, Horswell S, et al. Intratumor heterogeneity and branched evolution revealed by multiregion sequencing. N Engl J Med. 2012; 366:883-892.

117. Imhof A, Brunner P, Marincek N, et al. Response, survival, and long-term toxicity after therapy with the radiolabelled somatostatin analogue ${ }^{90} \mathrm{Y}$-DOTATOC in metastasized neuroendocrine cancers. J Clin Oncol. 2011;29:2416-2423.

118. Kwekkeboom DJ, de Herder WW, Kam BL, et al. Treatment with the radiolabeled somatostatin analog $\left[{ }^{177} \mathrm{Lu}-\mathrm{DOTA} 0, \mathrm{Tyr} 3\right]$ octreotate: toxicity, efficacy, and survival. J Clin Oncol. 2008;26:2124-2130.

119. Yao JC, Hassan M, Phan A, et al. One hundred years after "carcinoid": epidemiology of and prognostic factors for neuroendocrine tumors in 35,825 cases in the United States. J Clin Oncol. 2008;26:3063-3072.

120. Villard L, Romer A, Marincek N, et al. Cohort study of somatostatin-based radiopeptide therapy with $\left[{ }^{90} \mathrm{Y}\right.$-DOTA]-TOC versus $\left[{ }^{90} \mathrm{Y}-\mathrm{DOTA}\right]-\mathrm{TOC}$ plus $\left[{ }^{177} \mathrm{Lu}\right.$ DOTA]-TOC in neuroendocrine cancers. J Clin Oncol. 2012;30:1100-1106.

121. Kulke MH, Siu LL, Tepper JE, et al. Future directions in the treatment of neuroendocrine tumors: consensus report of the National Cancer Institute Neuroendocrine Tumor clinical trials planning meeting. J Clin Oncol. 2011;29:934-943.

122. Wang X, Fani M, Schulz S, Rivier J, Reubi JC, Maecker HR. Comprehensive evaluation of a somatostatin-based radiolabelled antagonist for diagnostic imaging and radionuclide therapy. Eur J Nucl Med Mol Imaging. 2012;39:1876-1885.

123. Ambrosini V, Fani M, Fanti S, Forrer F, Maecke HR. Radiopeptide imaging and therapy in Europe. J Nucl Med. 2011;52(suppl 2):42S-55S.

124. Barbet J, Chatal JF, Gauche F, Martino J. Which radionuclides will nuclear oncology need tomorrow? Eur J Nucl Med Mol Imaging. 2006;33:627-630.

125. Delbeke D, Segall HM. Status and trends in nuclear medicine in the US. J Nucl Med. 2011;52(suppl 2):24S-28S

126. Dondi M, Kashyap R, Paez D, et al. Trends in nuclear medicine in developing countries. J Nucl Med. 2011;52(suppl 2):16S-23S.

127. Graham MM, Menda Y. Radiopeptide imaging and therapy in the US. $J$ Nucl Med. 2011;52(suppl 2):56S-63S

128. Josephson L, Rudin M. Barriers to clinical translation with diagnostic drugs. J Nucl Med. 2013;54:329-332.

129. Schelbert HR. Nuclear medicine at a crossroads. J Nucl Med. 2011;52(suppl 2): $10 \mathrm{~S}-15 \mathrm{~S}$.

130. Schwaiger M, Wester HJ. How many PET tracers do we need? J Nucl Med. 2011;52(suppl 2):36S-41S.

131. Zimmermann RG. Industrial constraints in the selection of radionuclides and the development of new radiopharmaceuticals. World J Nucl Med. 2008;7:126-134.

132. Zimmermann RG. Why are investors not interested in my radiotracer? The industrial and regulatory constraints in the development of radiopharmaceuticals. Nucl Med Biol. 2013;40:155-166. 